\title{
A Feedback Interpretation of the Doyle-Fuller-Newman Lithium-ion Battery Model
}

\author{
R. Drummond, A. M. Bizeray, D. A. Howey and S. R. Duncan
}

\begin{abstract}
The Doyle-Fuller-Newman 'psuedo-2D' electrochemical model of a lithium ion battery is shown to have a feedback structure when electric-double layer effects are included. This opens the possibility for the model to be rigorously analysed using input-output systems theory. Several immediate consequences of the feedback structure are discussed, including observability and well-posedness issues, and an application to reduced order modelling is described.
\end{abstract}

Index Terms-Li-ion batteries , Nonlinear Systems, Energy Storage, Absolute Stability.

\section{INTRODUCTION}

A formulation of the classic Doyle-Fuller-Newman (DFN) electrochemical model for a lithium ion battery $[10,14,30]$ that includes a feedback structure and a statespace is proposed. The formulation is derived by incorporating fast double-layer capacitance dynamics into the model [31]. Recognising the inherent feedback structure of the nonlinear battery model opens the possibility for its analysis using input-output systems theory [9, 19]. A key feature of the model analysis is that it is robust, as it does not rely on the exact knowledge of the model nonlinearities such as the open-circuit potential (OCP) curves. As such, this formulation could allow a "generalised" analysis which would be suitable for all instances of the passive nonlinearities. The focus of the paper is to facilitate the use of the underlying DFN model by considering the structure in its model equations, not by proposing new electrochemistry. It is hoped that the results developed here will enable the DFN model to be used for wider applications, such as has been observed with the single particle model [8], and will reveal the key features of the model to promote its development and to understand its predictions about the internal electrochemistry.

Lithium ion batteries are a near ubiquitous energy storage technology that combine excellent power and energy density into one device. This has led to their successful application in many areas, including hybrid vehicles, personal electronics and grid storage. However, these batteries still suffer from several limitations, including the onset of ageing after several hundred charge cycles, stability issues

R. Drummond, A. M. Bizeray, D. A. Howey and S. R. Duncan are with the Department of Engineering Science, University of Oxford, 17 Parks Road, OX1 3PJ, Oxford, United Kingdom, Email: \{ross.drummond, adrien.bizeray, david.howey, stephen.duncan\}@ eng.ox.ac.uk.

This work was supported by the UK Engineering and Physical Research Council (EPSRC) through grant EP/005411/1-"Structured electrodes for improved energy storage". leading to thermal runaway and uncertainty in the stateof-charge estimation. To overcome these issues, methods from control theory, such as parameter estimation and observer design, have been developed [7]. Central to the successful application of these methods is an underlying model suitable for the application.

This paper is concerned with electrochemical models that use conservation and diffusion relationships to describe the various chemical reactions and ionic flows occurring within the battery [7]. Typically, these models describe effects such as the intercalation of lithium ions in the solid particles and ionic diffusion in the electrolyte. The added detail of current and potential spatial distributions within the battery from these models can be exploited to create improved battery management systems (BMS) that will allow batteries to be used more effectively [8]. Compared to other models, such as equivalent circuits, these models are also more suitable for design purposes, allowing novel batteries to be developed using fewer experiments. A benchmark electrochemical Li-ion battery model is the Doyle-Fuller-Newman model considered in this paper $[10,14,30]$. This model has seen extensive development and is regarded as being relatively accurate, provided the correct parameters are used [30].

The cost of increased model fidelity is complexity, with electrochemical models generally being in the form of a set of nonlinear partial differential equations coupled with algebraic constraints. Consequently, to apply these models in real time, such as in a battery management system, simplified versions are often used. These include the single particle model (SPM) and its many variants, including electrolyte dynamics [29] and temperature effects [16, 35]. Whilst making the model analysis tractable, these simplified methods often lose or obscure the description of the underlying electrochemistry that they describe and lose accuracy at high C-rate charging. An example where this becomes an issue is in the optimal design of electrodes with structure [17], where resolving the question on how to adapt the lumped particles to characterise the heterogeneous electrode structures is not clear. In this context, the aim of this paper is to address the open issue of developing an analytical framework for the analysis of the full DFN model.

\section{Contribution}

A formulation of the DFN model is proposed that can be interpreted using feedback. The key element of the 


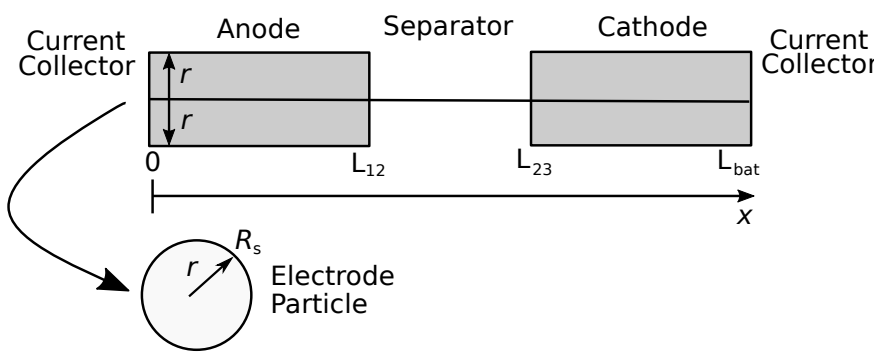

Figure 1: Domain geometry of the DFN model. Electrode particles are treated as being cylindrical in shape, with the spatial co-ordinate $x$ traversing both electrodes and the separator. Within each electrode there is a circular particle at each point $x$, with the co-ordinate $r$ going from the particle centre to the perimeter. This figure shows a $2 \mathrm{D}$ projection of the domain as well as a typical electrode particle.

formulation is the inclusion of double layer capacitance dynamics within the DFN model [31]. This formulation allows the DFN model to be rigorously analysed using systems theoretic concepts without the need for significant simplifying assumptions. A feature of the proposed analysis is that it is robust, being applicable when the nonlinearities (such as the OCP curves) are unknown. This is due to the consideration of the model as a Lurie system that can be analysed from an input-output perspective [9]. Some immediate consequences of this formulation are discussed, including issues regarding observability of the electrolyte concentration, the well-posedness of the model and its non-dimensional form. As an application of the model formulation, a method to reduce the spatially discretised model's dimension using balancing is proposed. This nonlinear model order reduction scheme retains the nonlinearities (including the reaction rate expression and the OCP curves) within the reduced order system by exploiting the feedback structure.

\section{Doyle-Fuller-Newman Model}

This section introduces the DFN Li-ion battery model $[10,14]$ which is reformulated in the subsequent sections. A detailed description of the model can be found in [30] and the various terms in the model are described in Table I. For the sake of presentation, we may drop the parameter subscripts for their domain association.

The geometry of the model domain is described in Figure 1, with the model often referred to as a pseudo twodimensional (P2D) model. This is because it is formed of a macro-scale co-ordinate $x$ accounting for ion transport from one current collector to the other as well as a microscale co-ordinate $r$ for the intercalation of lithium ions into the particles. It is assumed that at each point in space $x$ there is also a particle (giving the second dimension). The model variables are the concentration of ions in the electrolyte $c_{e}(x, t)$ and the solid particles $c_{s}(x, r, t)$, electrode current $i_{s}(x, t)$, electrolyte current $i_{e}(x, t)$, potential in the

\begin{tabular}{|c|c|c|}
\hline \multicolumn{3}{|c|}{ Spatial Variables } \\
\hline$x$ & Battery spatial variable. & $\mathrm{m}$ \\
\hline$r$ & Particle spatial variable. & $\mathrm{m}$ \\
\hline \multicolumn{3}{|c|}{ Variables } \\
\hline$c_{s}(x, r, t)$ & Particle Li concentration. & $\mathrm{mol} \mathrm{m}^{-3}$. \\
\hline$c_{s}^{\text {surf }}(x, t)$ & Li surface concentration. & $\mathrm{mol} \mathrm{m}^{-3}$. \\
\hline$c_{e}(x, t)$ & Li electrolyte concentration. & $\mathrm{mol} \mathrm{m}^{-3}$. \\
\hline$u_{s}(x, r, t)$ & Transformed concentration. & $\mathrm{mol} \mathrm{m}^{-2}$. \\
\hline$u_{s}^{\text {surf }}(x, t)$ & Transformed surface conc. & $\mathrm{mol} \mathrm{m}^{-2}$. \\
\hline$\phi_{s}(x, t)$ & Potential in the solid. & $\mathrm{V}$ \\
\hline$\phi_{e}(x, t)$ & Potential in the electrolyte. & $\mathrm{V}$. \\
\hline$\phi_{d l}(x, t)$ & $\phi_{s}(x, t)-\phi_{e}(x, t)$ & V. \\
\hline$i_{s}(x, t)$ & Current in the solid. & $\mathrm{A} \mathrm{m}^{-2}$ \\
\hline$i_{e}(x, t)$ & Current in the electrolyte. & $\mathrm{A} \mathrm{m}^{-2}$. \\
\hline$j(x, t)$ & Reaction rate. & $\mathrm{A} \mathrm{m}^{-3}$. \\
\hline$\eta(x, t)$ & Overpotential. & V. \\
\hline$U\left(u_{s}^{\text {surf }}\right)$ & Open circuit potential. & V. \\
\hline$i(t)$ & Applied current density. & $\mathrm{A} \mathrm{m}^{-2}$. \\
\hline$V(t)$ & Measured Voltage & V. \\
\hline \multicolumn{3}{|c|}{ Parameters } \\
\hline$c_{s}^{\max }$ & Max. Li particle concentration. & $\mathrm{mol} \mathrm{m}^{-3}$. \\
\hline$D_{s}$ & Particle dffusion coefficient. & $\mathrm{m}^{2} \mathrm{~s}^{-1}$ \\
\hline$R_{s}$ & Spherical radius of the particles. & $\mathrm{m}$ \\
\hline$L_{k}$ & Length. & $\mathrm{m}$. \\
\hline$L_{\text {bat }}$ & Total length of the battery. & $\mathrm{m}$. \\
\hline$\epsilon_{\mathrm{e}}$ & Porosity coefficient. & \\
\hline$b$ & Bruggeman coefficient. & \\
\hline$D_{\mathrm{e}}^{\mathrm{eff}}$ & Effective diffusion coefficient. & $\mathrm{m}^{2} \mathrm{~s}^{-1}$ \\
\hline$a_{s}$ & Specific Interfacial area. & $\mathrm{m}^{-1}$ \\
\hline$t_{+}$ & Transference number. & \\
\hline $\mathcal{F}$ & Faraday's constant. & $\mathrm{C} \mathrm{mol}^{-1}$. \\
\hline$\alpha_{c} / \alpha_{c}$ & Anode/Cathode transfer coefs. & \\
\hline$R$ & Universal gas Constant. & $\mathrm{mol}^{-1} \mathrm{~K}^{-1}$ \\
\hline$T$ & Temperature. & $\mathrm{K}$. \\
\hline$k$ & Exchange Rate Parameter. & $\mathrm{m}^{2.5} \mathrm{~mol}^{-0.5} \mathrm{~s}^{-1}$ \\
\hline$\sigma^{\text {eff }}$ & Effective electrode conductivity. & $\mathrm{S} \mathrm{m}^{-1}$ \\
\hline$\kappa^{\mathrm{eff}}$ & Effective electrolyte conductivity. & $\mathrm{S} \mathrm{m}^{-1}$ \\
\hline$R_{c t c}$ & Contact resistance. & $\Omega$ \\
\hline \multicolumn{3}{|c|}{ Sub and super scripts } \\
\hline 1 & Denotes anode parameters. & \\
\hline 2 & Denotes separator parameters. & \\
\hline 3 & Denotes cathode parameters. & \\
\hline 12 & Anode/separator boundary. & \\
\hline 23 & Cathode/Separator boundary. & \\
\hline
\end{tabular}

Table I: Nomenclature of the DFN model.

electrolyte $\phi_{e}(x, t)$ and in the solid $\phi_{s}(x, t)$ as well as the current $I(t)$ and voltage $V(t)$.

Lithium intercalation in the particles is modeled by a spherical diffusion equation

$$
\frac{\partial c_{\mathrm{s}}(x, r, t)}{\partial t}=\frac{1}{r^{2}} \frac{\partial}{\partial r}\left(r^{2} D_{\mathrm{s}} \frac{\partial c_{\mathrm{s}}(x, r, t)}{\partial r}\right),
$$

subject to the flux boundary conditions,

$$
\frac{\partial c_{\mathrm{s}}(x, 0, t)}{\partial r}=0 \quad \text { and } \quad D_{\mathrm{s}} \frac{\partial c_{\mathrm{s}}\left(x, R_{\mathrm{s}}, t\right)}{\partial r}=-j(x, t)
$$


The concentration at the surface of the particle plays an important role in the transfer of ions between the particles and the electrolyte and is denoted by $c_{s}^{\text {surf }}(x, t)=c_{s}\left(x, R_{s}, t\right)$. Under the change of variables $u_{s}(x, r, t)=r c_{s}(x, r, t)$, this spherical diffusion equation becomes

$$
\frac{\partial u_{s}(x, r, t)}{\partial t}=D_{s} \frac{\partial^{2} u_{s}(x, r, t)}{\partial r^{2}}
$$

subject to $u_{s}(x, 0, t)=0$ and

$$
\frac{1}{R_{s}} \frac{\partial u_{s}\left(x, R_{\mathrm{s}}, t\right)}{\partial r}-\frac{\left.u_{s}\left(x, R_{\mathrm{s}}, t\right)\right)}{R_{s}^{2}}=\frac{-j(x, t))}{D_{s}} .
$$

The value of $u_{s}(x, r, t)$ at the particle surface is denoted by $u_{s}^{\text {surf }}(x, t)=u_{s}\left(x, R_{s}, t\right)$.

Electroneutrality is assumed, implying that the concentration of positive $c_{+}(x, t)$ and negative ions $c_{-}(x, t)$ in the solution are the same, that is $c_{+}(x, t)=c_{-}(x, t)=c_{e}(x, t)$. Ion transport in the electrodes is governed by

$\left.\epsilon_{\mathrm{e}} \frac{\partial c_{\mathrm{e}}(x, t)}{\partial t}=\frac{\partial}{\partial x}\left(\epsilon_{\mathrm{e}} D_{\mathrm{e}}^{\mathrm{eff}}\left(c_{\mathrm{e}}(x, t)\right) \frac{\partial c_{\mathrm{e}}}{\partial x}\right)+a_{\mathrm{s}}\left(1-t_{+}\right) j(x, t)\right)$,

with the effective Fickian diffusivity given by $D_{\mathrm{e}}^{\mathrm{eff}}=D_{\mathrm{e}} \epsilon_{\mathrm{e}}^{b}$, $b$ being the Bruggeman coefficient accounting for porosity effects, and $c_{e}(x, t)$ subject to homogeneous flux boundary conditions on both sides. The actual concentration considered is the volume averaged concentration $\epsilon_{e} c_{e}$. In the separator, $j(x, t)=0$, with the ions diffusing according to

$$
\epsilon_{\mathrm{e}} \frac{\partial c_{\mathrm{e}}(x, t)}{\partial t}=\frac{\partial}{\partial x}\left(\epsilon_{\mathrm{e}} D_{\mathrm{e}}^{\mathrm{eff}}\left(c_{\mathrm{e}}(x, t)\right) \frac{\partial c_{\mathrm{e}}}{\partial x}\right) .
$$

The boundary conditions of $c_{e}(x, t)$ are $\partial c_{e}(x, t) / \partial x=0$ at the boundary between the current collector and electrode and $\epsilon_{\mathrm{e} 1 / 3} D_{\mathrm{e} 1 / 3}^{\mathrm{eff}} \partial c_{\mathrm{e}}(x, t) / \partial x=\epsilon_{\mathrm{e} 2} D_{\mathrm{e} 2}^{\mathrm{eff}} \partial c_{\mathrm{e}}(x, t) / \partial x$ at the boundary of the separator and the electrodes. Here the subscript 2 refers to the value in separator while $1 / 3$ refers to either the value in the anode or cathode, depending upon the boundary.

The overpotential, the perturbed local electric potential relative to the open circuit potential, is

$$
\eta(x, t)=\phi_{\mathrm{s}}(x, t)-\phi_{\mathrm{e}}(x, t)-U\left(u_{\mathrm{s}}^{\mathrm{surf}}(x, t)\right),
$$

with open-circuit potential (OCP) as $U\left(u_{\mathrm{s}}^{\operatorname{surf}}(x, t)\right)$. The OCP is usually an experimentally fitted function of the stoichiometry $x_{\mathrm{s}}^{\mathrm{surf}}(x, t)=c_{\mathrm{s}}^{\mathrm{surf}}(x, t) / c_{\mathrm{s}}^{\max }$, which can be transformed into a function of $u_{s}^{\text {surf }}(x, t)$ via a change of variables.

Reaction rate kinetics at the boundary of the particles satisfy the Butler-Volmer equation

$j(x, t)=\frac{i_{0}(x, t)}{\mathcal{F}}\left[\exp \left(\frac{\alpha_{\mathrm{a}} \mathcal{F}}{R T} \eta(x, t)\right)-\exp \left(\frac{-\alpha_{\mathrm{c}} \mathcal{F}}{R T} \eta(x, t)\right)\right]$

where the exchange current density $i_{0}(x, t)$ is

$i_{0}(x, t)=k \mathcal{F}\left(c_{\mathrm{s}}^{\max }-c_{\mathrm{s}}^{\text {surf }}(x, t)\right)^{\alpha_{\mathrm{a}}}\left(c_{\mathrm{s}}^{\text {surf }}(x, t)\right)^{\alpha_{\mathrm{c}}}\left(c_{\mathrm{e}}(x, t)\right)^{\alpha_{\mathrm{a}}}$.

It is assumed that Ohm's law holds in the solid phase

$$
\frac{i_{\mathrm{s}}(x, t)}{\sigma^{\mathrm{eff}}}=-\frac{\partial \phi_{\mathrm{s}}(x, t)}{\partial x}
$$

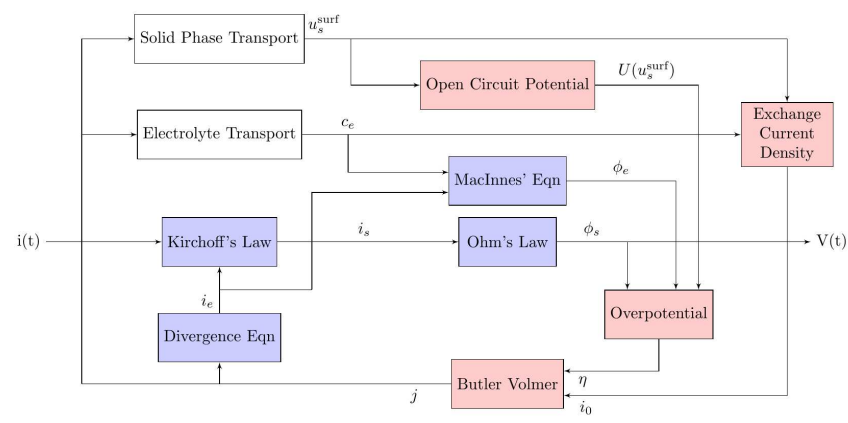

Figure 2: A block diagram representation of the standard formulation of the DFN model. Dynamical blocks are white, nonlinear functions are red and algebraic constraints are blue.

and MacInnes' equation holds in the liquid phase

$\frac{i_{\mathrm{e}}(x, t)}{\kappa^{\mathrm{eff}}\left(c_{e}(x, t)\right)}=-\frac{\partial \phi_{\mathrm{e}}(x, t)}{\partial x}+\frac{2\left(1-t_{+}\right) R T}{\mathcal{F}} \frac{\chi}{c_{\mathrm{T}} \bar{V}_{0}} \frac{\partial \ln c_{\mathrm{e}}(x, t)}{\partial x}$

with the effective ionic conductivity given by $\kappa^{\mathrm{eff}}=\kappa \epsilon_{\mathrm{e}}^{b}$. We make the approximation that the solvent concentration is only weakly dependent on concentration, which implies that $\chi /\left(c_{\mathrm{T}} \bar{V}_{0}\right) \approx 1$ and omit this term from the equations thereafter.

Current conservation is imposed by Kirchhoff's law

$$
i_{\mathrm{S}}(x, t)+i_{\mathrm{e}}(x, t)=i(t)
$$

with $i_{\mathrm{e}}=0$ at the current collectors and $i_{e}=i(t)$ at the electrode separator interfaces, as well as in the separator domain.

The divergence of the ionic current is related to the reaction rate by

$$
\frac{\partial i_{\mathrm{e}}(x, t)}{\partial x}=a_{\mathrm{s}} \mathcal{F} j(x, t)
$$

with the specific interfacial surface area given by $a_{\mathrm{s}}=$ $3 \epsilon_{\mathrm{s}} / R_{\mathrm{s}}$.

Voltage is given by:

$$
V(t)=\phi_{\mathrm{s}}\left(L_{b a t}, t\right)-\phi_{\mathrm{s}}(0, t)-R_{\mathrm{ctc}} i
$$

with $R_{\text {ctc }}$ the contact resistance at the currentcollector/electrode interfaces, given in its normalised form in $\Omega \mathrm{cm}^{2}$. The combination of these terms within the DFN model is shown in Figure 2, which highlights how each component interacts with the other.

\section{Doyle-Fuller-Newman Model with Double LAYER CAPACITANCE Dynamics}

The main results of the paper are contained within this section where the DFN model is augmented to include double layer capacitance effects. This augmented form of the model is then shown to lead to a feedback interpretation which is defined by a state-space model. 


\section{A. Model Formulation}

A double layer is a charge separating region formed at the interface between a charged electrode and the electrolyte [26]. Across this layer, the separation of charge between the electrode and the ions in the electrolyte leads to a capacitance, which is exploited by supercapacitors [26].

The inclusion of double-layer capacitance models in Liion batteries models has been comprehensively studied and in this paper, the model proposed by Ong and Newman in [31] is adopted. Here, double layer effects are included by augmenting the divergence equation (13) to

$$
a C \frac{\partial \phi_{d l}(x, t)}{\partial t}=\frac{\partial i_{e}(x, t)}{\partial x}-a_{s} F j(x, t)
$$

where $\phi_{d l}(x, t)=\phi_{s}(x, t)-\phi_{e}(x, t)$ is the potential difference across the double layer. This is the only equation that is adapted from the standard DFN model given in Section II. Other double layer models that have been developed for Li-ion batteries include [24] and [27]. The double layer potential $\phi_{d l}(x, t)$ satisfies the boundary conditions

$$
\frac{\partial \phi_{d l}(x, t)}{\partial x}=-\frac{i(t)}{\sigma^{\mathrm{eff}}}
$$

at the current collector/electrode boundary and

$$
\frac{\partial \phi_{d l}(x, t)}{\partial x}=\frac{i(t)}{\kappa^{\mathrm{eff}}}+\frac{K}{\kappa^{\mathrm{eff}}} \frac{\partial \ln c_{e}(x, t)}{\partial x}
$$

at the electrode/separator boundary. $K=\frac{2\left(1-t_{+}\right) R T}{\mathcal{F}} \frac{\chi}{c_{\mathrm{T}} \bar{V}_{0}}$ is introduced to simplify the notation. These boundary condition are obtained by noting that all the current is carried in the solid phase at the current collector boundary $i_{e}=0$ and by the electrolyte in the separator $i_{e}=i(t)$.

In most implementations of the DFN model, double layer effects are neglected as these dynamics are typically much faster than those of the other dynamical phenomenon occurring within the battery. In particular, the time constant of the double layer $\left(\tau=L^{2} a C \sigma \kappa /(\sigma+\kappa)\right)$ is typically $\approx 10^{-3} \mathrm{~s}[31]$ while that associated with the intercalation of lithium into the particles $\left(\tau=R_{s}^{2} / D_{s}\right)$ is typically $\approx 10^{3} \mathrm{~s}$ [4]. The intercalation is then the rate limiting step in this system, which explains the relative accuracy of the SPM for non-aggressive charging. However, it is argued in this section, that the inclusion of these fast dynamics greatly changes the structure of the battery model, permitting it to be analysed using input-output systems theory [9].

With double layer capacitance effects included, a statespace of $\mathrm{x}=\left\{c_{e}(x, t), u_{s}(x, r, t), \phi_{d l}(x, t)\right\}$ is proposed as these variables are associated with dynamics in (3), (5) and (15). The currents in the solid $i_{s}(x, t)$ and the electrolyte $i_{e}(x, t)$ represent auxiliary variables that have to be removed if the model is to be given a concise statespace form. To achieve this, it is noted that $i_{e}(x, t)=$ $i-i_{s}(x, t)=i+\sigma^{\text {eff } \frac{\partial \phi_{s}(x, t)}{\partial x}}$ and that by adding and subtracting $\kappa^{\text {eff }} \partial \phi_{s}(x, t) / \partial x$ from the RHS of the expression for $i_{e}(x, t)$ in $(11)$, then it can be equivalently expressed as

$$
\begin{aligned}
i_{e}(x, t)= & \left(\frac{\sigma^{\mathrm{eff}} \kappa^{\mathrm{eff}}}{\sigma^{\mathrm{eff}}+\kappa^{\mathrm{eff}}}\right) \frac{\partial \phi_{d l}(x, t)}{\partial x} \\
& +\left(\frac{1}{\sigma^{\mathrm{eff}}+\kappa^{\mathrm{eff}}}\right) i(t)+\left(\frac{K \sigma^{\mathrm{eff}}}{\sigma^{\mathrm{eff}}+\kappa^{\mathrm{eff}}}\right) \frac{\partial}{\partial x} \ln \left(c_{e}(x, t)\right) .
\end{aligned}
$$

The spatial derivative of electrolyte current is then

$$
\begin{aligned}
\frac{\partial i_{e}(x, t)}{\partial x}= & \left(\frac{\sigma^{\mathrm{eff}} \kappa^{\mathrm{eff}}}{\sigma+\kappa^{\mathrm{eff}}}\right) \frac{\partial^{2} \phi_{d l}(x, t)}{\partial^{2} x} \\
& +\left(\frac{K \sigma^{\mathrm{eff}}}{\sigma^{\mathrm{eff}}+\kappa^{\mathrm{eff}}}\right) \frac{\partial^{2}}{\partial x^{2}} \ln \left(c_{e}(x, t)\right)
\end{aligned}
$$

Using this expression, the DFN model can be written solely in terms of the state-space $\mathrm{x}$ as

$$
\begin{aligned}
\epsilon_{e} \frac{\partial c_{e}(x, t)}{\partial t} & =\epsilon_{e} D_{e}^{\mathrm{eff}} \frac{\partial^{2} c_{e}(x, t)}{\partial x^{2}}+a_{\mathrm{s}}\left(1-t_{+}\right) j(x, t) \\
\frac{\partial u_{s}(r, t)}{\partial t} & =D_{s} \frac{\partial^{2} u_{s}(r, t)}{\partial r^{2}} \\
a C \frac{\partial \phi_{d l}(x, t)}{\partial t} & =\left(\frac{\kappa^{\mathrm{eff}} \sigma^{\mathrm{eff}}}{\sigma^{\mathrm{eff}}+\kappa^{\mathrm{eff}}}\right) \frac{\partial^{2} \phi_{d l}(x, t)}{\partial x^{2}} \\
& -a_{s} F j(x, t)+\left(\frac{K \sigma^{\mathrm{eff}}}{\sigma^{\mathrm{eff}}+\kappa^{\mathrm{eff}}}\right) \frac{\partial^{2}}{\partial x^{2}} \ln \left(c_{e}(x, t)\right)
\end{aligned}
$$

Here, we have assumed that $D_{e}$ and $\kappa^{\text {eff }}$ are independent of $c_{e}$, which greatly simplifies the analysis of the proceeding sections as it permits the feedback form. However, if these variables are allowed to vary with $c_{e}(x, t)$, then the model should be interpreted in terms of linear parameter varying systems [6]. The system (20) is defined entirely by operators acting on the state-space and has eliminated the need for algebraic variables such as $i_{s}(x, t)$ and $i_{e}(x, t)$. The dynamics in the separator region remain unchanged from (6).

The essence of this formulation is that the inclusion of the double layer forces the spatio-temporal variables to evolve along a manifold of feasible solutions, while in the original form of the DFN model, the algebraic constraints ensure that the solutions remain on the manifold.

\section{B. Re-centering the Variables}

A change of co-ordinates is proposed to re-centre the nonlinearities around the origin, allowing the model to be expressed in terms of feedback signals. To achieve this, the electrolyte concentration is defined as $c_{e}(x, t)=$ $\tilde{c}(x, t)+c_{0}$ where $\tilde{c}(x, t)$ is the deviation of the concentration away from the static equilibrium $c_{0}$. The negative of the OCP is defined as $U^{-}\left(u_{s}^{\operatorname{surf}}(x, t)\right)=-U\left(u_{s}^{\text {surf }}(x, t)\right)$ and which is also re-centred around the origin by $U^{-}\left(u_{s}^{\operatorname{surf}}(x, t)\right)=U_{0}^{-}+\tilde{U}^{-}\left(u_{s}^{\text {surf }}(x, t)\right)$ where $\tilde{U}^{-}(0)=0$. Similarly, the potential difference across the double layer is re-centred according to $\phi_{d l}(x, t)=\tilde{\phi}_{d l}(x, t)-U_{0}^{-}$. 
The dynamics (20) defined around these new co-ordinates are

$$
\begin{aligned}
& \epsilon_{e} \frac{\partial \tilde{c}_{e}(x, t)}{\partial t}=\epsilon_{e} D_{e}^{\mathrm{eff}} \frac{\partial^{2} \tilde{c}_{e}(x, t)}{\partial x^{2}}+a_{\mathrm{s}}\left(1-t_{+}\right) j(x, t) \\
& \frac{\partial u_{s}(r, t)}{\partial t}=D_{s} \frac{\partial^{2} u_{s}(r, t)}{\partial r^{2}} \\
& a C \frac{\partial \tilde{\phi}_{d l}(x, t)}{\partial t}=\left(\frac{\kappa^{\mathrm{eff}} \sigma^{\mathrm{eff}}}{\sigma^{\mathrm{eff}}+\kappa^{\mathrm{eff}}}\right) \frac{\partial^{2} \tilde{\phi}_{d l}(x, t)}{\partial x^{2}} \\
&-a_{s} F j(x, t)+\left(\frac{K \sigma^{\mathrm{eff}}}{\sigma^{\mathrm{eff}}+\kappa^{\mathrm{eff}}}\right) \frac{\partial^{2}}{\partial x^{2}} \ln \left(1+\tilde{c}_{e}(x, t) / c_{0}\right) .
\end{aligned}
$$

with the overpotential defined as

$$
\eta=\tilde{\phi}_{d l}(x, t)+\tilde{U}^{-}\left(u_{s}^{\mathrm{surf}}(x, t)\right) .
$$

These new variables are subject to the same boundary conditions as in their untransformed cases.

\section{A Non-Dimensional Form}

A non-dimensionalised form of the model is now given, with the non-dimensional spatial lengths as $\bar{x}=x / L_{b a t} \in$ $[0,1]$ and $\bar{r}=r / R_{s} \in[0,1]$. The non-dimensional form of the dynamics is

$$
\begin{aligned}
\epsilon_{e} \frac{\partial \tilde{c}_{e}(\bar{x}, t)}{\partial t} & =\frac{\epsilon_{e} D_{e}^{\mathrm{eff}}}{L_{b a t}^{2}} \frac{\partial^{2} \tilde{c}_{e}(\bar{x}, t)}{\partial \bar{x}^{2}}+a_{\mathrm{s}}\left(1-t_{+}\right) j(\bar{x}, t) \\
\frac{\partial u_{s}(\bar{x}, \bar{r}, t)}{\partial t} & =\frac{D_{s}}{R_{s}^{2}} \frac{\partial^{2} u_{s}(\bar{x}, \bar{r}, t)}{\partial \bar{r}^{2}} \\
a C \frac{\partial \tilde{\phi}_{d l}(\bar{x}, t)}{\partial t} & =\frac{1}{L_{b a t}^{2}}\left(\frac{\kappa^{\mathrm{eff}} \sigma^{\mathrm{eff}}}{\sigma^{\mathrm{eff}}+\kappa^{\mathrm{eff}}}\right) \frac{\partial^{2} \tilde{\phi}_{d l}(\bar{x}, t)}{\partial \bar{x}^{2}} \\
-a_{s} F j(\bar{x}, t) & +\frac{1}{L_{b a t}^{2}}\left(\frac{K \sigma^{\mathrm{eff}}}{\sigma^{\mathrm{eff}}+\kappa^{\mathrm{eff}}}\right) \frac{\partial^{2}}{\partial \bar{x}^{2}} \ln \left(1+\tilde{c}_{e}(\bar{x}, t) / c_{0}\right)
\end{aligned}
$$

The non-dimensionalised boundary conditions can be easily obtained from before. Normalised diffusion coefficients can then be extracted

$$
\tau_{u_{s}}=\frac{D_{s}}{R_{s}^{2}}, \tau_{c_{e}}=\frac{D_{e}^{\mathrm{eff}}}{L_{b a t}^{2}}, \tau_{\phi_{d l}}=\left(\frac{\kappa^{\mathrm{eff}} \sigma^{\mathrm{eff}}}{L_{b a t}^{2} a C\left(\sigma^{\mathrm{eff}}+\kappa^{\mathrm{eff}}\right)}\right),
$$

where, typically, $\tau_{u_{s}} \ll \tau_{c_{e}} \ll \tau_{\phi_{d l}}$, with $\tau_{u_{s}} \approx 1 \times$ $10^{-4} \mathrm{~s}^{-1}, \tau_{c_{e}} \approx 1 \times 10^{-1} \mathrm{~s}^{-1}$ and $\tau_{\phi_{d l}} \approx 1 \times 10^{4} \mathrm{~s}^{-1}$.

\section{Voltage Expression}

The use of a state-space involving the potential difference across the double layer $\phi_{d l}(x, t)$, instead of $\phi_{s}(x, t)$ and $\phi_{e}(x, t)$ individually, complicates the expression for the voltage, which is defined in (14) by the difference in the potentials in the solid phase between the two current collectors. The following is used to express $V(t)$ as a function of the new state-space.

$$
\begin{aligned}
& V(t)=\phi_{s}\left(L_{\mathrm{bat}}, t\right)-\phi_{s}(0, t)-R_{\mathrm{ctc}} i \\
& =\phi_{d l}\left(L_{\mathrm{bat}}, t\right)-\phi_{d l}(0, t)+\phi_{e}\left(L_{\mathrm{bat}}, t\right)-\phi_{e}(0, t)-R_{\mathrm{ctc}} i \\
& =\phi_{d l}\left(L_{\mathrm{bat}}, t\right)-\phi_{d l}(0, t)+\int_{0}^{L_{\mathrm{bat}}} \frac{\partial \phi_{e}(x, t)}{\partial x} d x-R_{\mathrm{ctc}} i
\end{aligned}
$$

Substituting the expression for $\partial \phi_{e}(x, t) / \partial x$ from MacInnes' equation (11) gives

$$
\begin{aligned}
& V(t)=\phi_{d l}\left(L_{b a t}, t\right)-\phi_{d l}(0, t) \\
& +\int_{0}^{L_{b a t}} \frac{-i_{e}(x, t)}{\kappa^{\mathrm{eff}}}+\frac{K}{\kappa^{\mathrm{eff}}} \frac{\partial \ln \left(1+\tilde{c}_{e}(x, t) / c_{0}\right)}{\partial x} d x-R_{\mathrm{ctc}} i .
\end{aligned}
$$

Finally, using (18) to define $i_{e}(x, t)$,

$V(t)=\tilde{\phi}_{d l}\left(L_{\text {bat }}, t\right)-\tilde{\phi}_{d l}(0, t)$

$-\frac{\sigma_{1}^{\mathrm{eff}}}{\kappa_{1}^{\mathrm{eff}}+\sigma_{1}^{\mathrm{eff}}}\left(\tilde{\phi}_{d l}\left(L_{12}, t\right)-\tilde{\phi}_{d l}(0, t)\right)$

$-\frac{\sigma_{3}^{\text {eff }}}{\kappa_{3}^{\text {eff }}+\sigma_{3}^{\text {eff }}}\left(\tilde{\phi}_{d l}\left(L_{\text {bat }}, t\right)-\tilde{\phi}_{d l}\left(L_{23}, t\right)\right)$

$-\left(\frac{1}{\kappa_{1}^{\mathrm{eff}}\left(\kappa_{1}^{\mathrm{eff}}+\sigma_{1}^{\mathrm{eff}}\right)}+\frac{1}{\kappa_{3}^{\mathrm{eff}}\left(\kappa_{3}^{\mathrm{eff}}+\sigma_{3}^{\mathrm{eff}}\right)}+\frac{1}{\kappa_{2}^{\mathrm{eff}}}-R_{\mathrm{ctc}}\right) i(t)$

$-K\left(\left(\frac{\sigma_{1}^{\text {eff }}}{\kappa_{1}^{\text {eff }}\left(\kappa_{1}^{\text {eff }}+\sigma_{1}^{\text {eff }}\right)}-1\right)\left(\ln c_{e}\left(L_{12}, t\right)-\ln c_{e}(0, t)\right)\right.$

$-\frac{1}{\kappa_{2}^{\mathrm{eff}}}\left(\ln c_{e}\left(L_{23}, t\right)-\ln c_{e}\left(L_{12}, t\right)\right)$

$\left.-\left(\frac{\sigma_{3}^{\mathrm{eff}}}{\kappa_{3}^{\mathrm{eff}}\left(\kappa_{3} \mathrm{eff}+\sigma_{3}^{\mathrm{eff}}\right)}-1\right)\left(\ln c_{e}\left(L_{\mathrm{bat}}, t\right)-\ln c_{e}\left(L_{23}, t\right)\right)\right)$.

Referring to Table I, the subscripts 1,2 and 3 respectively refer to the parameters in the anode, separator and cathode and the lengths $x=0, x=L_{12}, x=L_{23}, x=L_{b a t}$ refer to the anode/current collector boundary, the anode/separator boundary, the separator/cathode boundary and the cathode/current collector boundary.

\section{Nonlinear Feedback Systems}

The notion of a feedback interpretation of a nonlinear system is described in this section [9], with the DFN model of (23) being considered a Lurie system [19,6]. As described below, this class of system is represented by the feedback interconnection of a linear system with static, (locally) sector bounded nonlinearities of a certain class. Even though these systems are nonlinear, they can be analysed using many of the techniques from linear systems, making their analysis feasible.

\section{A. Sector Bounded Nonlinearities and Lurie Systems}

Consider the class of nonlinear functions $\psi(y): \mathcal{Y} \subseteq$ $\mathbb{R}^{m} \rightarrow \mathbb{R}^{m}$ satisfying the following properties: zero at zero $\psi(0)=0$, decentralised

$$
\psi(y)=\left[\psi_{1}\left(y_{1}\right), \psi_{2}\left(y_{2}\right), \ldots, \psi_{n_{p}}\left(y_{m}\right)\right]^{T},
$$


and locally sector bounded

$$
\frac{\psi_{j}\left(y_{j}\right)}{y_{j}} \in\left[0, \tau_{j}\right] \quad \forall y \in \mathcal{Y} \subseteq \mathbb{R}^{m} .
$$

These nonlinearities represent sign-preserving operators acting on $\mathcal{Y}$ and in the language of circuits, they represent passive nonlinear components. An example of a nonlinear function that satisfies these conditions is given in Figure 3 .

A Lurie system is a particular class of nonlinear system with the structure

$$
\left\{\begin{array}{l}
\dot{\mathrm{x}}=\mathcal{A} \mathrm{x}+\mathcal{B} \psi(y)+\mathcal{B}_{i} i(t) \\
y=\mathcal{C}_{\mathrm{x}}+\mathcal{D} \psi(y)+\mathcal{D}_{i} i(t) \\
V=\mathcal{C}_{z} \mathrm{x}+\mathcal{D}_{z} \psi(y)+\mathcal{D}_{z i} i(t)
\end{array}\right.
$$

with $\left\{\mathcal{A}, \mathcal{B}, \mathcal{B}_{i}, \mathcal{C}, \mathcal{D}, \mathcal{D}_{i}, \mathcal{C}_{z}, \mathcal{D}_{z}, \mathcal{D}_{z i}\right\}$ being linear operators. The nonlinear terms $\psi(y)$ enter the Lurie system in an affine manner in both the output signals $y$ and the dynamics. It is this affine nature that gives the Lurie system its feedback interpretation, with the typical feedback representation shown in Figure 4.

The outputs that the feedback nonlinearities act on are termed $y$ while the output of the system that is measured by a physical sensor is the voltage $V$. This distinction between the measured output $(V)$ and those defining the feedback loops $(y)$ is standard for Lurie systems [6] as it separates the signals used in the analysis and those that are measured. The current $i(t)$ represents an external disturbance while the nonlinearities represent feedback actions, with $\psi_{j}\left(y_{j}\right)=\frac{\psi_{j}\left(y_{j}\right)}{y_{j}} \cdot y_{j}$ being the classic notion of a feedback signal with nonlinear feedback gain $\frac{\psi_{j}\left(y_{j}\right)}{y_{j}} \in\left[0, \tau_{j}\right] \quad \forall y \in \mathcal{Y} \subseteq \mathbb{R}^{n_{p}}$. It is stressed that the input and output signals, $y$ and $\psi(y)$, which define the feedback structure of the Lurie system do not need to be physically measurable.

The dynamical analysis of Lurie systems can be framed in either the time domain or the frequency domain, with time domain results typically involving the construction of Lyapunov functions [19]. A key feature of the analysis of these systems is that it is robust, in the sense that stability guarantees, and other system properties, hold for all nonlinearities satisfying the sector conditions (26). It is this generality that has led to the extensive interest in these systems since their introduction over 50 years ago. Robustness is a key benefit offered by this approach, as the model nonlinearities, such as the OCP curve, are only approximately known at a given point in time, and may change during a cell's lifetime due to ageing [3].

\section{Feedback Interpretation of the DFN Model}

It is now shown that the DFN model of (23) has the feedback structured of a Lurie system. The model has four nonlinearities, each of which are shown to satisfy the sector conditions (26). These four nonlinearities are plotted in figure 5 .

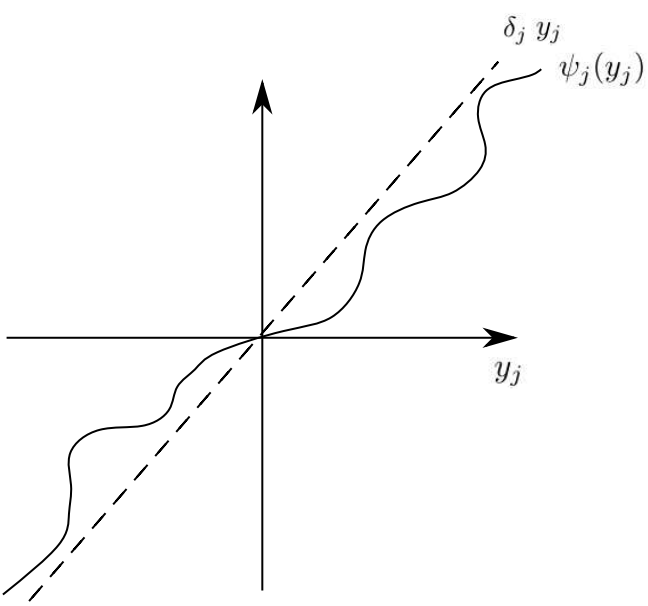

Figure 3: A decentralised nonlinearity $\psi_{j}\left(y_{j}\right)$ that satisfies the sector conditions.

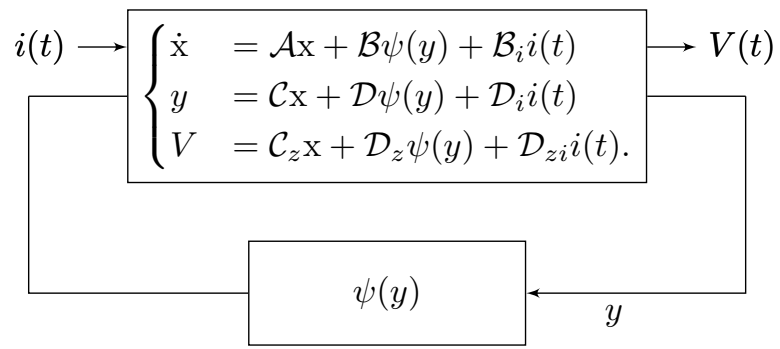

Figure 4: A typical representation of a Lurie system expressed in terms of feedback.

\section{A. Open Circuit Potential}

The first nonlinearity is the OCP curve $U^{-}\left(u_{s}^{\text {surf }}(x, t)\right)$ which appears in the expression for the overpotential $\eta(x, t)$ in $(22)$. The presence of $U^{-}\left(u_{s}^{\text {surf }}\right)$ in the expression for the reaction rate $j(x, t)$, causes $j(x, t)$ to be a nested nonlinearity, being nested in $U^{-}\left(u_{s}^{\text {surf }}(x, t)\right)$. This is equivalent to stating there exists the term $\mathcal{D}$ in the state-space feedback matrices of (27). Due to its assumed monotonicity, the OCP curve is sector bounded from each point on the curve. The condition $\psi(0)=0$ is achieved for the OCP curve by the change of co-ordinates from $U\left(u_{s}^{\text {surf }}(x, t)\right) \rightarrow U^{-}\left(u_{s}^{\text {surf }}(x, t)\right)$. As the OCP curve is only locally defined, the analysis must be contained to some finite region of the state-space such that $y \in \mathcal{Y}_{0} \subseteq \mathcal{Y} \subseteq$ $\mathbb{R}^{m}$, and as such, the geometrical properties of Lyapunov functions [19] represent more suitable tools for analysis than the functional analysis approach, which is posed in the frequency domain [9].

\section{B. Reaction Rate}

The second nonlinearity is the chemical reaction rate $j(x, t)$, appearing in (8). This nonlinearity is not decentralised due to the polynomial ion exchange rate current $i_{0}(x, t)$ of (9). This lack of decentralisation can be overcome by considering the representation $j(x, t)=i_{0}(x, t) j_{0}(x, t)$ where $j_{0}=$ 


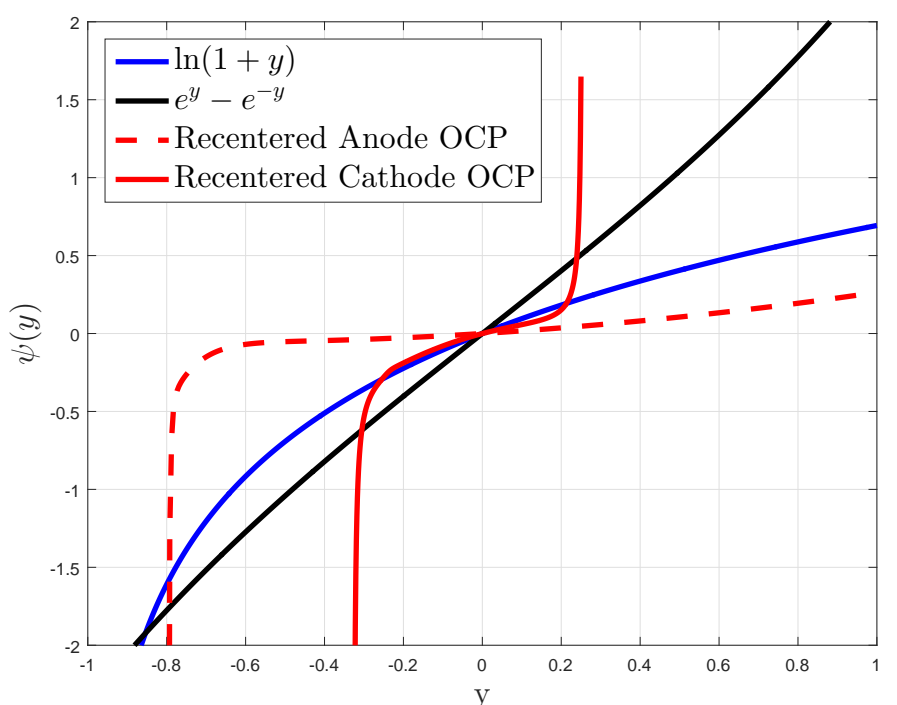

Figure 5: The various nonlinearities of the DFN model including the OCP curves, the exponential term of the Butler-Volmer equation and the logarithmic term from MacInnes' equation. After the re-centering of the statespace variables, these nonlinearities all satisfy the sector conditions.

$\frac{1}{\mathcal{F}}\left[\exp \left(\frac{\alpha_{\mathrm{a}} \mathcal{F}}{R T} \eta(x, t)\right)-\exp \left(\frac{-\alpha_{\mathrm{c}} \mathcal{F}}{R T} \eta(x, t)\right)\right]$ and is both decentralised and sector bounded. The mapping $j_{0}(x, t) \rightarrow$ $j(x, t)$ involves the multiplication by a positive term $i_{0}(x, t) \geq 0$, which can be treated as a multiplicative disturbance $i_{0}(x, t)=\beta(x, t) \geq 0$. Abstracting $i_{0}(x, t)$ to simply be a positive gain in this manner means that a decentralised mapping from $\eta(x, t) \rightarrow j(x, t)$ is obtained. The key aspect is that $i_{0}(x, t)$ is always positive and so can be considered to be a sign preserving gain acting on $j_{0}(x, t)$. The abstraction of $i_{0}(x, t)$ to simply be a positive multiplier, rather than a polynomial operator on the states as in (9), may lead to a conservative analysis, which could be alleviated by considering a smaller local domain, giving tighter bounds on $i_{0}(x, t)=\beta(x, t) \in[0, \bar{\beta}]$.

\section{Logarithmic term}

The third nonlinearity is the shifted logarithm stemming from MacInnes' equation $\ln \left(1+\tilde{c}_{e}(x, t) / c_{0}\right)$. This nonlinearity lies in the sector $[0, \infty)$ but crucially is only locally defined [38].

\section{Feedback form}

With these nonlinearities, the feedback form of the DFN model has the structure of figure 6 , where $\mathrm{x}=$ $\left\{c_{e}(x, t), u_{s}(r, t), \phi_{d l}(x, t)\right\}$ is the state, $\psi=\{j(x, t), \ln (1+$ $\left.\left.c_{e}(x, t) / c_{0}\right), U\left(u_{s}^{\text {surf }}(x, t)\right)\right\}$ is the nonlinear feedback and $y=\left\{\eta(x, t), u_{s}^{\text {surf }}, c_{e}(x, t)\right\}$ are the output signals.

\section{Model Implementation: Spatial Discretisation}

For implementation, the partial differential equations (PDEs) of the DFN model with capacitance effects in-

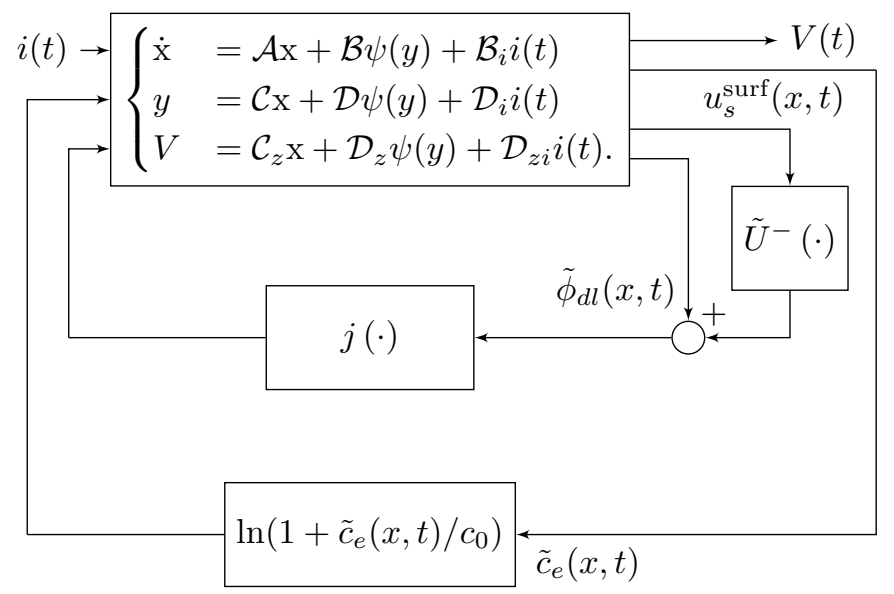

Figure 6: Feedback interconnection of the DFN model with double layer effects included.

cluded (23) are discretised in space to give a finite dimensional realisation. In this paper, Chebyshev polynomials of the first kind are used as interpolation functions of the solutions to the PDEs, with the spatial domain being a grid of Chebyshev nodes [37]. Details of this discretisation procedure for the DFN model are given in [5], with a similar procedure applied to an electrochemical supercapacitor model in [11]. By expanding the solution using "global" interpolation functions such as Chebyshev polynomials, the discretised system can be both low-order and accurate when compared against other discretisation methods, such as finite-difference. The discretised form of the model equations (23) is

$$
\begin{aligned}
{\left[\begin{array}{c}
\dot{\tilde{\boldsymbol{c}}}_{\boldsymbol{e}} \\
\dot{\boldsymbol{u}}_{\boldsymbol{s}} \\
\dot{\tilde{\boldsymbol{\phi}}}_{\boldsymbol{d l}}
\end{array}\right]=} & {\left[\begin{array}{ccc}
\boldsymbol{D}_{c e} & 0 & 0 \\
0 & \boldsymbol{D}_{u_{s}} & 0 \\
0 & 0 & \boldsymbol{D}_{\phi_{d l}}
\end{array}\right]\left[\begin{array}{c}
\tilde{\boldsymbol{c}}_{\boldsymbol{e}} \\
\boldsymbol{u}_{s} \\
\tilde{\boldsymbol{\phi}}_{\boldsymbol{d l}}
\end{array}\right] } \\
+ & {\left[\begin{array}{ccc}
\boldsymbol{a}_{\mathbf{1}} & 0 & 0 \\
\boldsymbol{b}_{\mathbf{2}} & 0 & 0 \\
\boldsymbol{b}_{\mathbf{3}} & \boldsymbol{b}_{\mathbf{4}} & 0
\end{array}\right]\left[\begin{array}{c}
\boldsymbol{j}(\boldsymbol{\eta}) \\
\ln \left(\mathbf{1}+\tilde{\boldsymbol{c}}_{\boldsymbol{e}} / \boldsymbol{c}_{\mathbf{0}}\right) \\
\boldsymbol{U}\left(\boldsymbol{u}_{\boldsymbol{s}}^{\text {surf }}\right)
\end{array}\right]+\left[\begin{array}{c}
0 \\
0 \\
\boldsymbol{b}_{\boldsymbol{i}}
\end{array}\right] i(t) }
\end{aligned}
$$

where the boundary conditions of the states have been incorporated and bold face is used to denote finite dimensional vectors and matrices. The matrices $\boldsymbol{D}_{\zeta}$ are known as discretisation matrices. The discrete version of the feedback system outputs are

$$
\begin{aligned}
{\left[\begin{array}{c}
\boldsymbol{\eta} \\
\tilde{\boldsymbol{c}}_{\boldsymbol{e}} \\
\boldsymbol{u}_{\boldsymbol{s}}^{\text {surf }}
\end{array}\right] } & =\left[\begin{array}{ccc}
0 & 0 & \boldsymbol{c}_{1} \\
\boldsymbol{c}_{2} & 0 & 0 \\
0 & 0 & c_{3}
\end{array}\right]\left[\begin{array}{c}
\tilde{c}_{\boldsymbol{e}} \\
\boldsymbol{u}_{s} \\
\tilde{\phi}_{d l}
\end{array}\right] \\
+ & {\left[\begin{array}{ccc}
0 & 0 & \boldsymbol{d}_{1} \\
0 & 0 & 0 \\
d_{2} & 0 & 0
\end{array}\right]\left[\begin{array}{c}
\boldsymbol{j}(\boldsymbol{\eta}) \\
\ln \left(1+\tilde{\boldsymbol{c}}_{\boldsymbol{e}} / \boldsymbol{c}_{\mathbf{0}}\right) \\
\boldsymbol{U}\left(\boldsymbol{u}_{\boldsymbol{s}}^{\text {surf }}\right)
\end{array}\right] }
\end{aligned}
$$

and the voltage is approximated by

$$
V(t)=\boldsymbol{C}_{z} \boldsymbol{x}+\boldsymbol{D}_{z} \boldsymbol{\psi}(\boldsymbol{y})+\boldsymbol{D}_{z i} i(t) .
$$


The associated finite dimensional Lurie system is

$$
\begin{cases}\dot{\mathbf{x}} & =\boldsymbol{A} \mathbf{x}+\boldsymbol{B}_{\boldsymbol{\psi}} \boldsymbol{\psi}(\boldsymbol{y})+\boldsymbol{B}_{\boldsymbol{i}} i(t) \\ \boldsymbol{y} & =\boldsymbol{C}_{\boldsymbol{y}} \mathbf{x}+\boldsymbol{D}_{\boldsymbol{y}} \boldsymbol{\psi}(\boldsymbol{y})+\boldsymbol{D}_{i} i(t) \\ V(t) & =\boldsymbol{C}_{\boldsymbol{z}} \mathbf{x}+\boldsymbol{D}_{\boldsymbol{z}} \boldsymbol{\psi}(\boldsymbol{y})+\boldsymbol{D}_{\boldsymbol{z} i} i(t) .\end{cases}
$$

where $\mathbf{x}=\left[\tilde{\boldsymbol{c}}_{\boldsymbol{e}}^{T}, \boldsymbol{u}_{s}^{T}, \tilde{\boldsymbol{\phi}}_{\boldsymbol{d l}}^{T}\right]^{T}, \boldsymbol{y}=\left[\boldsymbol{\eta}^{T}, \tilde{\boldsymbol{c}}_{\boldsymbol{e}}^{T}, \boldsymbol{u}_{\boldsymbol{s}}^{\text {surf } \boldsymbol{T}}\right]^{T}$ and $\boldsymbol{\psi}(\boldsymbol{y})=\left[\boldsymbol{j}(\boldsymbol{\eta})^{T}, \ln \left(\mathbf{1}+\tilde{\boldsymbol{c}}_{\boldsymbol{e}} / \boldsymbol{c}_{\mathbf{0}}\right)^{T}, \boldsymbol{U}\left(\boldsymbol{u}_{\boldsymbol{s}}^{\text {surf }}\right)^{T}\right]^{T}, \boldsymbol{A} \in \mathbb{R}^{n \times n}$, $\boldsymbol{B} \in \mathbb{R}^{n \times m}, \boldsymbol{B}_{\boldsymbol{i}} \in \mathbb{R}^{n \times 1}, \boldsymbol{C}_{\boldsymbol{y}} \in \mathbb{R}^{m \times n}, \boldsymbol{D}_{\boldsymbol{y}} \in \mathbb{R}^{m \times m}, \boldsymbol{D}_{\boldsymbol{i}} \in$ $\mathbb{R}^{m \times 1}, \boldsymbol{C}_{\boldsymbol{z}} \in \mathbb{R}^{1 \times n}, \boldsymbol{D}_{\boldsymbol{z}} \in \mathbb{R}^{1 \times m}$ and $\boldsymbol{D}_{\boldsymbol{z} \boldsymbol{i}} \in \mathbb{R}^{1 \times 1}$. These systems matrices are defined as

$$
\begin{aligned}
\boldsymbol{A} & =\left[\begin{array}{ccc}
\boldsymbol{D}_{c e} & 0 & 0 \\
0 & \boldsymbol{D}_{u_{s}} & 0 \\
0 & 0 & \boldsymbol{D}_{\phi_{d l}}
\end{array}\right], \\
\boldsymbol{B}_{\psi} & =\left[\begin{array}{lll}
\boldsymbol{a}_{\mathbf{1}} & 0 & 0 \\
\boldsymbol{b}_{\mathbf{2}} & 0 & 0 \\
\boldsymbol{b}_{\mathbf{3}} & \boldsymbol{b}_{\mathbf{4}} & 0
\end{array}\right], \boldsymbol{B}_{i}=\left[\begin{array}{c}
0 \\
0 \\
\boldsymbol{b}_{\boldsymbol{i}}
\end{array}\right], \\
\boldsymbol{C}_{\boldsymbol{y}} & =\left[\begin{array}{ccc}
0 & 0 & \boldsymbol{c}_{\mathbf{1}} \\
\boldsymbol{c}_{\mathbf{2}} & 0 & 0 \\
0 & 0 & \boldsymbol{c}_{\boldsymbol{3}}
\end{array}\right], \boldsymbol{C}_{\boldsymbol{z}}=\left[\begin{array}{lll}
\boldsymbol{c}_{z} & 0 & 0
\end{array}\right], \\
\boldsymbol{D}_{\boldsymbol{y}} & =\left[\begin{array}{ccc}
0 & 0 & \boldsymbol{d}_{\mathbf{1}} \\
0 & 0 & 0 \\
\boldsymbol{d}_{\mathbf{2}} & 0 & 0
\end{array}\right], \boldsymbol{D}_{i}=\left[\begin{array}{l}
0 \\
0 \\
0
\end{array}\right], \\
\boldsymbol{D}_{\boldsymbol{z}} & =\left[\begin{array}{lll}
0 & \boldsymbol{d}_{z} & 0
\end{array}\right], \boldsymbol{D}_{\boldsymbol{z i}}=\left[\boldsymbol{d}_{z i}\right]
\end{aligned}
$$

Even though no algebraic equations are present in the model dynamics (31), an implicit algebraic equation still exists due to the feed-through matrices $\boldsymbol{D}_{\boldsymbol{y}}$ in the output signals $\boldsymbol{y}$. More specifically, upon inspection of the boundary conditions (4) on $u_{s}^{\text {surf }}(x, t)$, it follows that $u_{s}^{\text {surf }}(x, t)$ is a function of $j(x, t)$, which is itself a function of $u_{s}^{\text {surf }}(x, t)$ via the nesting OCP curve $U\left(u_{s}^{\text {surf }}(x, t)\right)$. A circular argument then emerges on obtaining $u_{s}^{\text {surf }}(x, t)$ which must be resolved by solving an algebraic equation. The dynamics of the model used for simulation are then

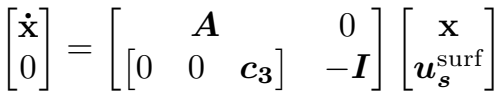

$$
\begin{aligned}
& +\left[\begin{array}{ccc}
\boldsymbol{d}_{\mathbf{2}} & 0 & 0
\end{array}\right] \boldsymbol{\psi}(\boldsymbol{y})+\left[\begin{array}{c}
\boldsymbol{B}_{i} \\
0
\end{array}\right] i(t)
\end{aligned}
$$

which is a differential algebraic equation (DAE) system. DAE systems can be challenging to simulate, however, the analysis can still be posed in terms of the feedback system (28).

Figures 7 compares the DFN model with and without double layer capacitance effects for a combined ARTEMIS drive cycle [1]. Shown in the figure are the two voltage responses, the voltage error between the two simulations, the state-of-charge $(S o C)$ in the anode and the applied current density. The model parameters were obtained from [5] and describe a battery with a lithium cobalt oxide cathode and a mesocarbon microbead anode with $1 \mathrm{M} \mathrm{LiPF}_{6}$ in propylene carbonate, ethylene carbonate and dimethyl carbonate (PC/EC/DMC) electrolyte. The capacitance value $a C=22.61 \times 10^{3} \mathrm{~F} \mathrm{~m}^{-2}$ from [31] was used and the ion exchange current $\left.i_{(} x, t\right)$ satisfied $(9)$ for these simulations. The error between the two simulations is plotted in Figure 7b, with the normalised error as defined using the RSME

$$
\left\|V_{\text {error }}\right\|=\sqrt{\frac{\sum_{k=1}^{N}\left(V_{k}^{D F N}-V_{k}^{D L}\right)^{2}}{N}}=0.0197 \mathrm{~V}
$$

where $N$ is the number of simulation time steps, $V_{k}^{D F N}$ is the $k^{\text {th }}$ element of the voltage vector from the simulation of the full DFN model whilst $V_{k}^{D L}$ is that with double layer effects included. This simulation reinforces the notion that accuracy improvements offered by including double layer capacitance effects can often be small (the difference in $S o C$ was less than $1 \%$ ), but these effects give a more complete system structure.

\section{Some ImplicAtions OF THE FORMUlAtion}

Several properties of the feedback DFN model which can be immediately obtained from its feedback interpretation are now discussed.

\section{A. Observability of Electrolyte Concentration}

One of the key applications for electrochemical based Li-ion battery models is observer design [7]. An observer is used to predict the internal state of the battery using only sensor information of the current and voltage. For a battery application, one of the main tasks of an observer is to obtain an accurate estimate of the state-of-charge, a property that reflects the remaining charge that can be drawn from the device. Typically, observers that are based on electrochemical models use the SPM due to its relative simplicity $[29,35,5]$. Critical to the performance of an observer is the "observability" of the system which for a nonlinear system can be regarded as a measure of how well the states can be reconstructed from voltage measurements (paraphrased from [40]). A state is unobservable if it does not affect the voltage, so that there is a fundamental limitation on obtaining an accurate estimate of these unobservable states.

Inspecting the model of (23), it follows that if $t_{+}=1$ and $i_{0}(x, t)$ is set to be independent of $c_{e}(x, t)$, then the electrolyte concentration is unobservable with regards to the voltage. This stems from the fact that the choice of transference number eliminates the logarithmic term from the dynamics (as $K=0$ ), with the dynamics for $c_{e}(x, t)$ then being completely hidden from the voltage sensor measurements. A rigorous observability analysis of the model could be implemented using the Lie derivative approach described in [40].

\section{B. Well-Posedness of the Feedback Loop}

An essential requirement for feedback systems is that they are well posed, i.e. the feedback admits a unique solution. The question of well-posedness is decoupled from that of stability, and is an essential feature for the existence of a system theoretic representation of the model [9]. 


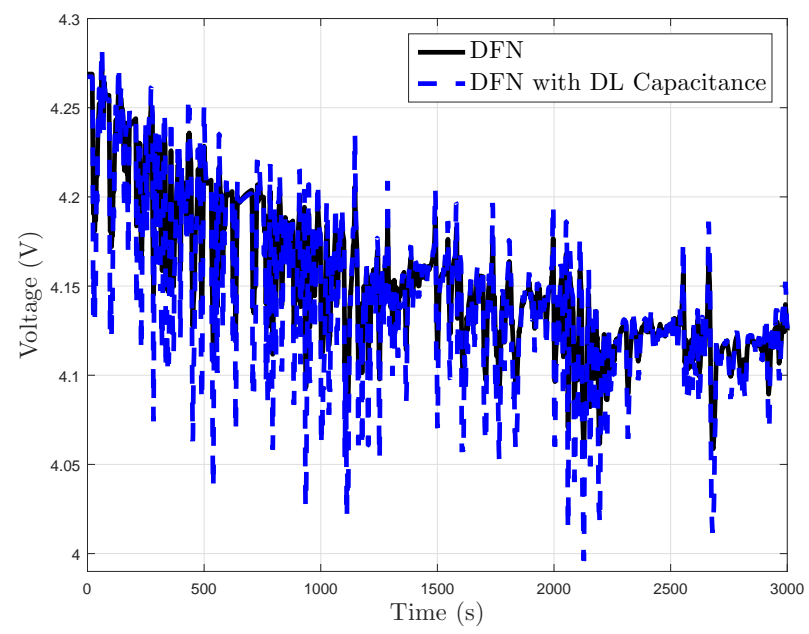

(a) Voltage responses.

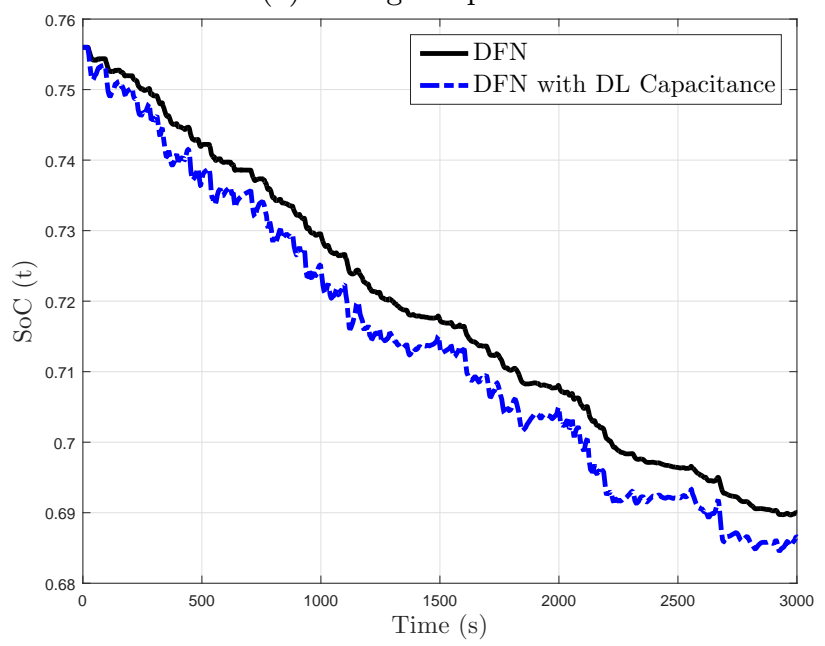

(c) Evolution of the state-of-charge.

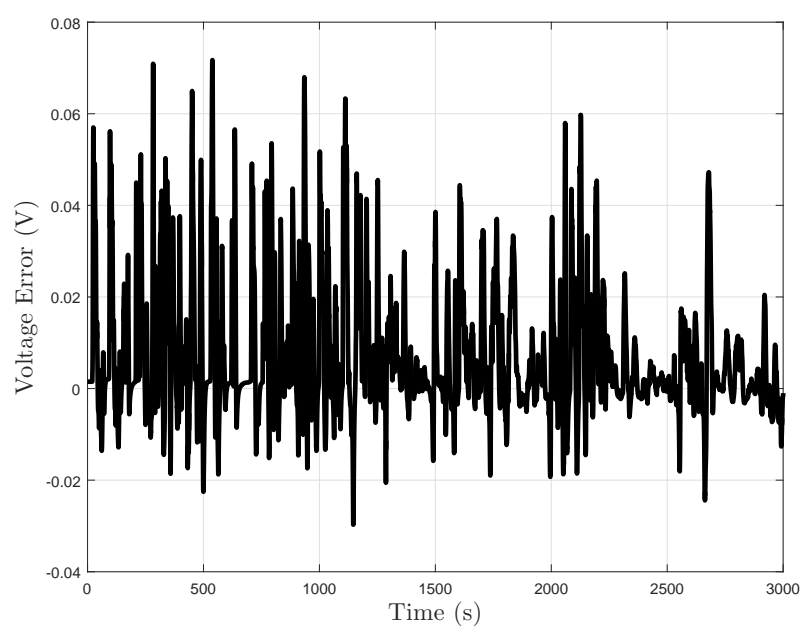

(b) Voltage error

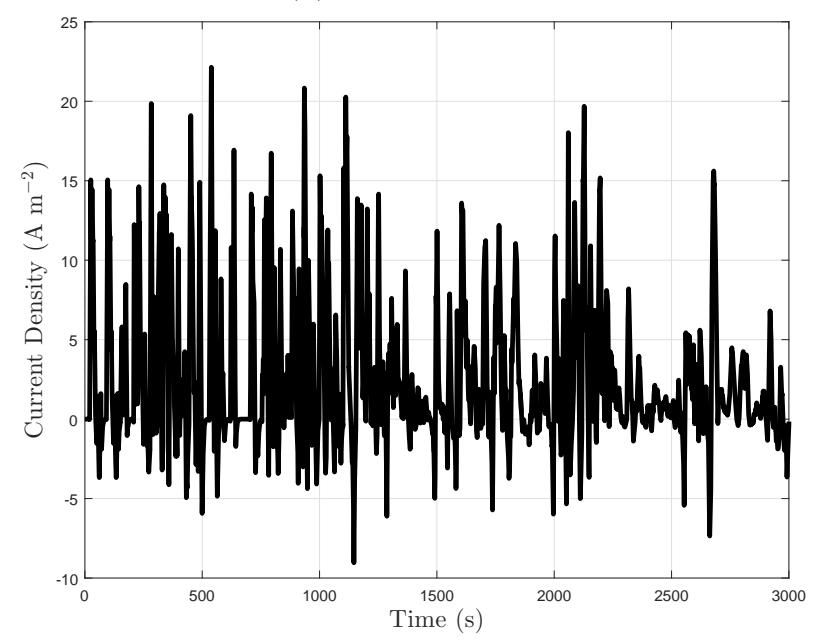

(d) Applied current density.

Figure 7: Simulations of the DFN model with and without double layer capacitance under the ARTEMIS drive cycle discharging current.

The well-posedness of a feedback loop containing a feedthrough term, as in (31), is guaranteed if it can be shown that there exists a unique solution to the implicit equation $\boldsymbol{F}(\boldsymbol{\mu}):=\boldsymbol{\mu}-\boldsymbol{D}_{\boldsymbol{y}} \boldsymbol{\psi}(\boldsymbol{\mu})$. Following [38], this condition can be equivalently stated as requiring the Jacobian of $\boldsymbol{F}(\boldsymbol{\mu})$ to belong to a compact and convex set of invertible matrices for almost all values of $\boldsymbol{\mu}$, which is satisfied if the following condition holds.

Proposition 1 (Well-posedness [38]): Define $\overline{\boldsymbol{\Gamma}}=\operatorname{diag}\left(\max \left(d \boldsymbol{\psi}_{\mathbf{1}}\left(\boldsymbol{y}_{\mathbf{1}}\right) / d \boldsymbol{y}_{\mathbf{1}}\right), \ldots, \max \left(d \boldsymbol{\psi}_{\boldsymbol{m}}(\boldsymbol{y},) / d \boldsymbol{y}_{\boldsymbol{m}} \boldsymbol{t}\right)\right)$ and $\underline{\boldsymbol{\Gamma}}=\operatorname{diag}\left(\min \left(d \boldsymbol{\psi}_{\mathbf{1}}\left(\boldsymbol{y}_{\mathbf{1}}\right) / d \boldsymbol{y}_{\mathbf{1}}\right), \ldots, \min \left(d \boldsymbol{\psi}_{\boldsymbol{m}}(\boldsymbol{y},) / d \boldsymbol{y}_{\boldsymbol{m}} \boldsymbol{t}\right)\right.$ The feedback system (28) is well posed if there exists a matrix $\boldsymbol{W} \in \mathbb{D}_{>0}^{m}$, with $\mathbb{D}_{>0}^{m}$ being the set of positive semi-definite matrices of dimension $m$, such that

$$
2 \boldsymbol{W}-H e\left(\boldsymbol{W}\left(\boldsymbol{I}-\boldsymbol{D}_{\boldsymbol{y}} \underline{\boldsymbol{\Gamma}}\right)^{-1} \boldsymbol{D}_{\boldsymbol{y}}(\overline{\boldsymbol{\Gamma}}-\underline{\boldsymbol{\Gamma}})\right)>0 .
$$

This condition was satisfied by the discretised model for $\underline{\boldsymbol{\Gamma}}=0$ and $\overline{\boldsymbol{\Gamma}}$ exceeding $10^{8}$, implying its well-posedness.

\section{ROBUST ANALYSIS OF PARAMETER VARIATIONS}

A main assumption of the formulation of the DFN model considered in Section II is that the model parameters, such as the electrolyte diffusion coefficient, remain constant during a charge. However, experimental data has shown that this assumption is violated for deep and high Crate charging, with temperature and concentration effects being highlighted as being particularly important. For instance, it is typically assumed that $\kappa$ varies linearly with concentration $c_{e}[39]$ while diffusivity follows an Arrhenius type relation with temperature [21, 36]. This section discusses how these parameter variations can also be included in the robust feedback framework.

A general setting is adopted to highlight how the analysis remains valid for parameter perturbations corresponding to different electrochemical phenomena. Consider a parameter $\theta(x, t)$ that is allowed to vary in both space and time and could represent the effective diffusion coefficient of the electrolyte for example. Expanding this parameter 
value around an equilibrium value $\theta_{0}$ gives

$$
\theta(x, t)=\theta_{0}+\tilde{\theta}(x, t) .
$$

If this parameter represents the diffusion coefficient of a species $w(x, t)$, such as for the electrolyte dynamics in (5), then the resulting diffusion equation becomes

$$
\begin{aligned}
\frac{\partial w}{\partial t} & =\frac{\partial}{\partial x}\left(\theta(x, t) \frac{\partial w}{\partial x}\right), \\
& =\theta_{0} \frac{\partial^{2} w}{\partial x^{2}}+\tilde{\theta}(x, t) \frac{\partial^{2} w}{\partial x^{2}}+\frac{\partial \tilde{\theta}(x, t)}{\partial x} \frac{\partial w}{\partial x}, \\
& =\theta_{0} \frac{\partial^{2} w}{\partial x^{2}}+\Gamma w .
\end{aligned}
$$

In such circumstances, the parameter variations are incorporated within the operator $\Gamma=\tilde{\theta}(x, t) \frac{\partial^{2}}{\partial x^{2}}+\frac{\partial \tilde{\theta}(x, t)}{\partial x} \frac{\partial}{\partial x}$.

This system then represents a linear-parameter varying system whose discretisation can be analysed using the methods of [28] for example. In such circumstances, the dynamics become

$$
\dot{\boldsymbol{w}}=A \boldsymbol{w}+\boldsymbol{\Gamma}(\boldsymbol{\theta}) \boldsymbol{w}
$$

with state $\boldsymbol{w} \in \mathbb{R}^{n_{w}}$, parameters $\boldsymbol{\theta} \in \mathbb{R}^{n_{\theta}}$ and with the operator $\Gamma$ becoming a vector $\Gamma \in \mathbb{R}^{n}$. If bounds on the variations of the parameters are known, then $\Gamma$ takes values within the compact set

$$
\boldsymbol{\Gamma}_{k} \in\left[\underline{\boldsymbol{\Gamma}}_{k}, \overline{\boldsymbol{\Gamma}}_{k}\right]
$$

There then exists $n_{w}$ sector conditions

$$
\left(\overline{\boldsymbol{\Gamma}}_{k} \boldsymbol{w}_{k}-\boldsymbol{\Gamma}_{k} \boldsymbol{w}_{k}\right)\left(\underline{\boldsymbol{\Gamma}}_{k} \boldsymbol{w}_{k}-\boldsymbol{\Gamma}_{k} \boldsymbol{w}_{k}\right) \geq 0
$$

from which one can then apply the same robust analysis as employed in Section V for the DFN model using absolute stability techniques. Using this approach, parameter variations, from temperature and concentration influences on the diffusion coefficient $D_{e}^{e f f}$, can then be incorporated into the analysis.

\section{Reduced Order DFN Model}

One of the main limitations of electrochemical models, such as the DFN model, is that the large number of states after spatial discretisation makes them unsuitable for online implementation within a battery management system and other control applications. Typically, the discretised DFN model has around 100 states, while online systems, in general, are suitable for models with less than 10 states [25]. To overcome this issue, reduced order versions of electrochemical models have been proposed in the literature, including the control orientated models of Smith et al. $[33,20,34,18]$ and the discrete time realisation algorithm of Plett et al. [32, 23, 22, 15]. All of these methods involve linearisation at some stage, and typically involve approximations of transfer functions.

A method for obtaining reduced order realisations of the feedback based DFN model that retains the nonlinearities is proposed here. The model order reduction is performed by exploiting the feedback structure of the system. The proposed approach can be considered as an approximation of a transfer function by a lower order form, but in this method, the transfer function is MIMO and involves all of the feedback nonlinearities discussed in section $\mathrm{V}$.

The model order reduction is based upon a balanced truncation of the linear state-space matrices $(\boldsymbol{A}, \boldsymbol{B}, \boldsymbol{C}, \boldsymbol{D})$, with these matrices characterising the Lurie system (31) with

$$
\boldsymbol{B}=\left[\begin{array}{ll}
\boldsymbol{B}_{\psi} & \boldsymbol{B}_{i}
\end{array}\right], \boldsymbol{C}=\left[\begin{array}{l}
\boldsymbol{C}_{\boldsymbol{y}} \\
\boldsymbol{C}_{z}
\end{array}\right], \boldsymbol{D}=\left[\begin{array}{cc}
\boldsymbol{D}_{\boldsymbol{y}} & \boldsymbol{D}_{i} \\
\boldsymbol{D}_{z} & \boldsymbol{D}_{z i}
\end{array}\right]
$$

The MIMO transfer function for this state-space realisation is $\boldsymbol{G}(s)=\boldsymbol{C}(s \boldsymbol{I}-\boldsymbol{A})^{-1} \boldsymbol{B}+\boldsymbol{D}$ which maps the extended inputs $\boldsymbol{u}_{\boldsymbol{e}}=$ $\left[\boldsymbol{j}(\boldsymbol{\eta})^{T}, \ln \left(1+\tilde{\boldsymbol{c}}_{\boldsymbol{e}} / c_{0}\right)^{T}, \boldsymbol{U}\left(\boldsymbol{u}_{\boldsymbol{s}}^{\text {surf }}\right)^{T}, i(t)\right]^{T}$ to the extended outputs $\boldsymbol{y}_{\boldsymbol{e}}=\left[\boldsymbol{\eta}^{T}, \tilde{\boldsymbol{c}}_{\boldsymbol{e}}^{T}, \boldsymbol{u}_{\boldsymbol{s}}^{\text {surf } T}, V(t)\right]^{T}$ in the frequency domain.

The balancing method is just one of many model order reduction methods but is popular due to its relative simplicity, its intuitive concept and its error bounds, see [13] for a comprehensive summary of the balancing method. The application of balancing to Lurie systems was developed in [2], where theoretical bounds on the accuracy of the reduced order models were given. Unfortunately, these bounds are not valid for the system considered here as the nonlinearities of the DFN model are only defined locally for $y \in \mathcal{Y}$, whereas a global analysis was considered in [2].

Consistent initial conditions $\boldsymbol{x}_{\boldsymbol{r}}(0)$ for the reduced order system can be obtained by noting that $\boldsymbol{y}_{e}$ should be the same for both the reduced and full order models. Defining $\boldsymbol{C}_{\boldsymbol{r}}$ as the reduced order form of the matrix $\boldsymbol{C}$, the reduced order initial condition $\boldsymbol{x}_{\boldsymbol{r}}(0)$ can be obtained from

$$
\boldsymbol{x}_{\mathbf{0}, \boldsymbol{r e d}}=\left(\boldsymbol{C}_{\boldsymbol{r}}^{T} \boldsymbol{C}_{\boldsymbol{r}}\right)^{-1}\left(\boldsymbol{C}_{\boldsymbol{r}}^{T} \boldsymbol{y}_{\boldsymbol{e}}(0)-\boldsymbol{D} \boldsymbol{u}_{\boldsymbol{e}}(0)\right)
$$

under the assumption that $\left(\boldsymbol{C}_{\boldsymbol{r}}^{T} \boldsymbol{C}_{\boldsymbol{r}}\right)^{-1}$ exists. Here, $\boldsymbol{y}_{\boldsymbol{e}}(0)$ and $\boldsymbol{u}_{\boldsymbol{e}}(0)$ are the values of the extended input and output signals of the full order model at time 0s.

An issue with applying the balancing method to reduce the order of the DFN model is that the matrix $\boldsymbol{A}$ is, in general, not Hurwitz as some of its eigenvalues are at the origin. To overcome this issue, an eigen-decomposition can be applied to the state-space matrices, with the model order reduction only being applied to the Hurwitz states. A similar procedure was adopted in [12] for supercapacitors. The circuit interpretation of the non-Hurwitz states is that they represent a set of capacitors connected in parallel which can be combined to form a single capacitor, so that these states can be represented by a single combined state. In this manner, a one state version of the DFN model with feedback can be obtained, with the dynamics of the single state being an integrator.

Using this model order reduction technique, the feedback structure of the model is retained, but the model states no longer have a physical interpretation; these states become mechanisms by which energy is transferred from the inputs to the output, and are a combination of the different physical phenomena present in the battery model. 


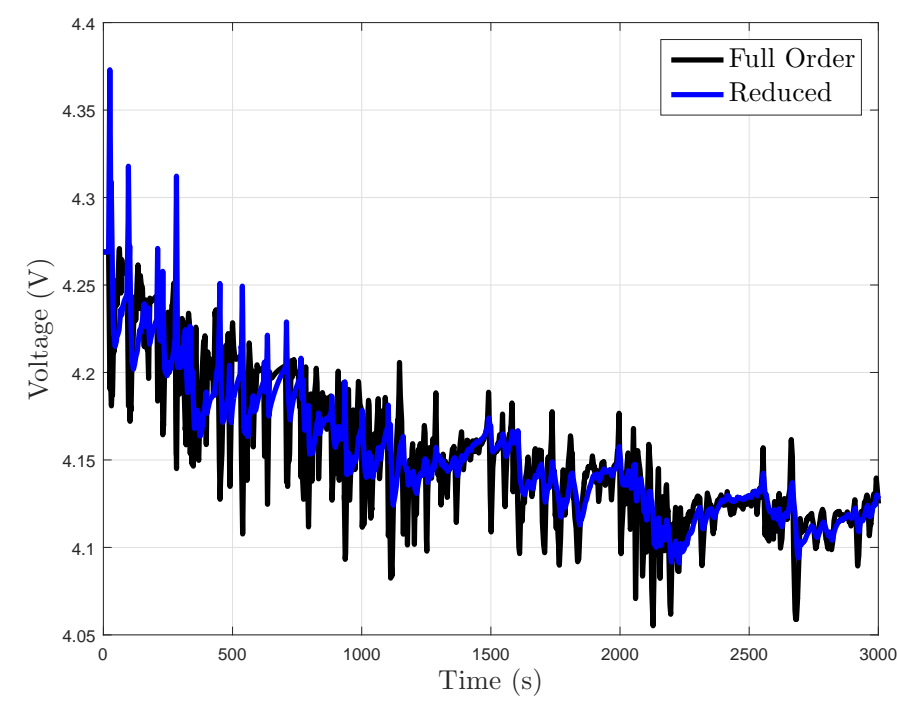

Figure 8: Responses of the full DFN model (28) and its reduced order form, with the reduced model only having one dynamics states, under the ARTEMIS drive cycle.

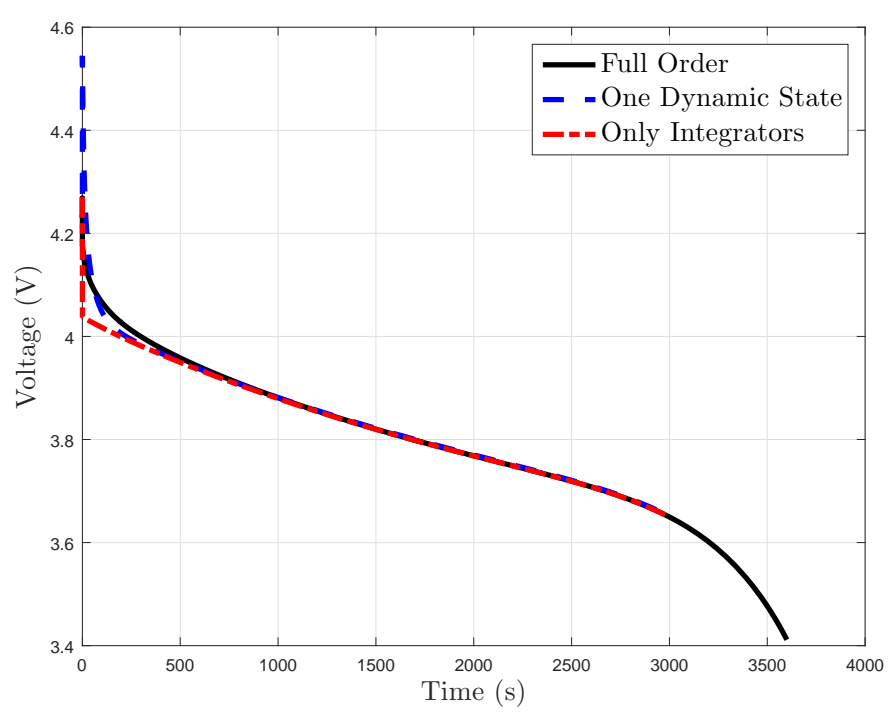

Figure 9: A constant current discharge, with current density at $20 \mathrm{~A} \mathrm{~m} \mathrm{~s}^{-2}$ for both the full order DFN model of (28), a reduced form with only one dynamic state and another with only integrators (no dynamic states).

The accuracy of the reduced order model is compared in Figures 8 and 9. In Figure 8, the full DFN model with double layer effects and varying $i_{0}(x, t)$ is simulated with the combined ARTEMIS drive cycle [1] against a reduced order model with only one dynamical state. In Figure 9 both the full order model, a reduced order model with only non-Hurwitz states, and a reduced model with the nonHurwitz states and one dynamic state, is discharged at a rate of $1 \mathrm{C}$. In general, the reduced order model captures the dynamical response of the full order model, especially after the initial transients of the simulations due to the initial conditions had passed. In the drive cycle simulation, after the initial transient phase, the reduced order model was found to be less responsive than the full order model. This is due to the balancing procedure eliminating the states with fast dynamics. In the constant current simulation, the reduced order models failed prematurely at $t \approx 3000$ s due to their states no longer resembling those of the full order model and leaving the region where the model nonlinearities are defined.

\section{Conclusions}

By including double-layer capacitance effects with the classic DFN electrochemical model for a lithium ion battery, the model was shown to exhibit a nonlinear feedback structure. This structure allows the model to be analysed rigorously using input-output systems theory. Exploiting this feedback structure leads to a deeper understanding of the model behaviour and could lead to observers and optimal charging profiles being designed for the DFN model. Some relevant consequences of the model formulation are discussed, including its non-dimensional form, its well-posedness and the observability of the electrolyte concentration. As an application, a novel reduced order form of the model is proposed that retains the nonlinearity.

\section{REFERENCES}

[1] M. André, "The ARTEMIS European driving cycles for measuring car pollutant emissions," Science of the total Environment, vol. 334, pp. 73-84, 2004.

[2] B. Besselink, N. van de Wouw, and H. Nijmeijer, "Model reduction for nonlinear systems with incremental gain or passivity properties," Automatica, vol. 49, no. 4, pp. 861-872, 2013.

[3] C. R. Birkl, M. R. Roberts, E. McTurk, P. G. Bruce, and D. A. Howey, "Degradation diagnostics for lithium ion cells," Journal of Power Sources, vol. 341, pp. 373-386, 2017.

[4] A. M. Bizeray, J.-H. Kim, S. R. Duncan, and D. A. Howey, "Identifiability and parameter estimation of the single particle lithium-ion battery model," IEEE Transactions on Control Systems Technology, no. 99, pp. 1-16, 2018.

[5] A. M. Bizeray, S. Zhao, S. R. Duncan, and D. A. Howey, "Lithium-ion battery thermal-electrochemical model-based state estimation using orthogonal collocation and a modified extended kalman filter," Journal of Power Sources, vol. 296, pp. 400-412, 2015.

[6] S. Boyd, L. El Ghaoui, E. Feron, and V. Balakrishnan, Linear matrix inequalities in system and control theory. SIAM, Philadelphia, PA, 1994.

[7] N. A. Chaturvedi, R. Klein, J. Christensen, J. Ahmed, and A. Kojic, "Algorithms for advanced batterymanagement systems," IEEE Control Systems, vol. 30, no. 3, pp. 49-68, 2010.

[8] — "Modeling, estimation, and control challenges for lithium-ion batteries," in Procs. of the American Control Conference. IEEE, 2010, pp. 1997-2002.

[9] C. A. Desoer and M. Vidyasagar, Feedback systems: input-output properties. SIAM, Philadelphia, PA, 2009. 
[10] M. Doyle, T. F. Fuller, and J. Newman, "Modeling of galvanostatic charge and discharge of the lithium/polymer/insertion cell," Journal of the Electrochemical Society, vol. 140, no. 6, pp. 1526-1533, 1993.

[11] R. Drummond, D. A. Howey, and S. R. Duncan, "Loworder mathematical modelling of electric double layer supercapacitors using spectral methods," Journal of Power Sources, vol. 277, pp. 317-328, 2015.

[12] R. Drummond, S. Zhao, D. A. Howey, and S. R. Duncan, "Circuit synthesis of electrochemical supercapacitor models," Journal of Energy Storage, vol. 10, pp. $48-55,2017$.

[13] G. E. Dullerud and F. Paganini, A course in robust control theory: a convex approach. Springer Science \& Business Media, New York, NY, 2013, vol. 36.

[14] T. F. Fuller, M. Doyle, and J. Newman, "Simulation and optimization of the dual lithium ion insertion cell," Journal of the Electrochemical Society, vol. 141, no. 1, pp. 1-10, 1994.

[15] K. Gopalakrishnan, T. Zhang, and G. J. Offer, "A fast, memory-efficient discrete-time realization algorithm for reduced-order li-ion battery models," Journal of Electrochemical Energy Conversion and Storage, vol. 14, no. 1, p. 011001, 2017.

[16] M. Guo, G. Sikha, and R. E. White, "Single-particle model for a lithium-ion cell: thermal behavior," Journal of The Electrochemical Society, vol. 158, no. 2, pp. A122-A132, 2011.

[17] C. Huang, N. P. Young, J. Zhang, H. J. Snaith, and P. S. Grant, "A two layer electrode structure for improved li ion diffusion and volumetric capacity in li ion batteries," Nano Energy, vol. 31, pp. 377-385, 2017.

[18] M. Jun, K. Smith, and P. Graf, "State-space representation of Li-ion battery porous electrode impedance model with balanced model reduction," Journal of Power Sources, vol. 273, pp. 1226-1236, 2015.

[19] H. K. Khalil, Nonlinear Systems. Prentice-Hall, New Jersey, 2009.

[20] G.-H. Kim, K. Smith, K.-J. Lee, S. Santhanagopalan, and A. Pesaran, "Multi-domain modeling of lithiumion batteries encompassing multi-physics in varied length scales," Journal of The Electrochemical Society, vol. 158, no. 8, pp. A955-A969, 2011.

[21] R. Klein, N. A. Chaturvedi, J. Christensen, J. Ahmed, R. Findeisen, and A. Kojic, "Electrochemical model based observer design for a lithium-ion battery," IEEE Transactions on Control Systems Technology, vol. 21, no. 2, pp. 289-301, 2013.

[22] J. L. Lee, A. Chemistruck, and G. L. Plett, "Discretetime realization of transcendental impedance models, with application to modeling spherical solid diffusion," Journal of Power Sources, vol. 206, pp. 367$377,2012$.

[23] — "One-dimensional physics-based reduced-order model of lithium-ion dynamics," Journal of Power Sources, vol. 220, pp. 430-448, 2012.
[24] N. Legrand, S. Raël, B. Knosp, M. Hinaje, P. Desprez, and F. Lapicque, "Including double-layer capacitance in lithium-ion battery mathematical models," Journal of Power Sources, vol. 251, pp. 370-378, 2014.

[25] L. Lu, X. Han, J. Li, J. Hua, and M. Ouyang, "A review on the key issues for lithium-ion battery management in electric vehicles," Journal of power sources, vol. 226, pp. 272-288, 2013.

[26] M. Lu, Supercapacitors: materials, systems and applications. Wiley-VCH Verlag GmbH and Co, Weinheim, Germany, 2013.

[27] J. Marcicki, A. Conlisk, and G. Rizzoni, "A lithiumion battery model including electrical double layer effects," Journal of Power Sources, vol. 251, pp. 157$169,2014$.

[28] J. Mohammadpour and C. W. Scherer, Control of linear parameter varying systems with applications. Springer Science \& Business Media, New York, NY, 2012.

[29] S. J. Moura, F. B. Argomedo, R. Klein, A. Mirtabatabaei, and M. Krstic, "Battery state estimation for a single particle model with electrolyte dynamics," IEEE Transactions on Control Systems Technology, vol. 25, no. 2, pp. 453-468, 2017.

[30] J. Newman and K. E. Thomas-Alyea, Electrochemical systems. John Wiley \& Sons, Honoken, NJ, 2012.

[31] I. J. Ong and J. Newman, "Double-layer capacitance in a dual lithium ion insertion cell," Journal of The Electrochemical Society, vol. 146, no. 12, pp. 43604365, 1999.

[32] A. Rodríguez and G. L. Plett, "Controls-oriented models of lithium-ion cells having blended electrodes. Part 2: Physics-based reduced-order models," Journal of Energy Storage, vol. 11, pp. 219-236, 2017.

[33] K. A. Smith, C. D. Rahn, and C.-Y. Wang, "Control oriented 1D electrochemical model of lithium ion battery," Energy Conversion and management, vol. 48, no. 9, pp. 2565-2578, 2007.

[34] — , "Model order reduction of 1D diffusion systems via residue grouping," Journal of Dynamic Systems, Measurement, and Control, vol. 130, no. 1, p. 011012, 2008.

[35] S.-X. Tang, L. Camacho-Solorio, Y. Wang, and M. Krstic, "State-of-charge estimation from a thermal-electrochemical model of lithium-ion batteries," Automatica, vol. 83, pp. 206-219, 2017.

[36] —, "State-of-charge estimation from a thermalelectrochemical model of lithium-ion batteries," $\mathrm{Au}$ tomatica, vol. 83, pp. 206-219, 2017.

[37] L. N. Trefethen, Spectral methods in MATLAB. SIAM, Philadelphia, PA, 2000.

[38] G. Valmorbida, R. Drummond, and S. Duncan, "Positivity conditions of Lyapunov functions for systems with slope restricted nonlinearities," in Proc. of the American Control Conference. IEEE, 2016, pp. 258263.

[39] M. W. Verbrugge and P. Liu, "Microstructural analysis and mathematical modeling of electric double- 
layer supercapacitors," Journal of the Electrochemical Society, vol. 152, no. 5, pp. D79-D87, 2005.
[40] M. Vidyasagar, Nonlinear systems analysis. SIAM, Philadelphia, PA, 2002. 